\title{
Mechanical thrombectomy in patients with cervical artery dissection and stroke in the anterior or posterior circulation - a multicenter analysis from the German Stroke Registry
}

Ludwig Schlemm ${ }^{1,2,3} \mathbb{D}$, Regina von Rennenberg ${ }^{1,2,3,4}$, Eberhard Siebert ${ }^{2,5}$, Georg Bohner ${ }^{2,5}$, Fabian Flottmann ${ }^{6}$, Gabor C. Petzold ${ }^{7,8}$, Götz Thomalla9 ${ }^{9}$, Matthias Endres ${ }^{1,2,3,4,10}$, Christian H. Nolte $1,2,3,4,10,11^{*}$ and for the GSR-ET investigators

\begin{abstract}
Background: Cervical artery dissection (CAD) is a rare cause of acute ischemic stroke (AIS) with large vessel occlusion (LVO) and may constitute a challenge for mechanical thrombectomy (MT). We compared procedural characteristics, reperfusion rates, and clinical outcome in AIS patients undergoing MT with and without CAD.

Methods: We performed a pre-specified analysis of patients registered within the German Stroke Registry, a prospectively maintained multicenter registry of consecutive patients with AIS patients treated by MT. Procedural characteristics included time periods and additional application of medication.

Results: Of 2589 patients, 62 (2.4\%) were diagnosed with CAD. CAD patients were younger, had lower rates of known vascular risk factors and larger baseline stroke volumes. MT in CAD patients took significantly longer (median [IQR] groin-puncture-to-flow restoration time: 98 [67-136] versus 70 [45-100] minutes; $p<0.001$ ) and more often required use of intra-arterial medication (34.4\% versus 15.6\%; $p<0.001$ ). Reperfusion success (modified Treatment in Cerebral Infarction score $2 \mathrm{~b}-3: \mathbf{8 5 . 2 \%}$ versus $83.3 \%, p=0.690$ ) and favorable functional outcome after 3 months (modified Rankin Scale score $\leq 2: 70.9 \%$ versus $36.4 \%$, adjusted $p=0.086$ ) did not differ significantly between patients with and without CAD. The latter findings held true for both CAD in the anterior and posterior circulation. Conclusion: CAD in AIS requiring MT is rare. MT in patients with CAD constitutes a particular procedural challenge, but still achieves favorable radiological and functional outcomes in most patients. Our data provide indirect evidence that MT is of clinical benefit in patients with AIS due to LVO and CAD.
\end{abstract}

Keywords: Ischemic stroke, Mechanical thrombectomy, Acute therapy, Dissection, Registry

\footnotetext{
* Correspondence: christian.nolte@charite.de

'Klinik und Hochschulambulanz für Neurologie, Charité - Universitätsmedizin

Berlin, corporate member of Freie Universität Berlin, Humboldt-Universität zu Berlin, Berlin, Germany

${ }^{2}$ Berlin Institute of Health (BIH), Berlin, Germany

Full list of author information is available at the end of the article
}

(c) The Author(s). 2021 Open Access This article is licensed under a Creative Commons Attribution 4.0 International License, which permits use, sharing, adaptation, distribution and reproduction in any medium or format, as long as you give appropriate credit to the original author(s) and the source, provide a link to the Creative Commons licence, and indicate if changes were made. The images or other third party material in this article are included in the article's Creative Commons licence, unless indicated otherwise in a credit line to the material. If material is not included in the article's Creative Commons licence and your intended use is not permitted by statutory regulation or exceeds the permitted use, you will need to obtain permission directly from the copyright holder. To view a copy of this licence, visit http://creativecommons.org/licenses/by/4.0/. 


\section{Introduction \\ Background}

Mechanical thrombectomy (MT) improves survival and reduces disability in patients with acute ischemic stroke (AIS) due to large vessel occlusion (LVO) of the anterior circulation $[1,2]$. The majority of LVO originates from cardioembolic or atherosclerotic etiologies [3-6]. Stroke etiology may modify the endovascular treatment effect [5].

Cervical artery dissection (CAD) represents a rare cause of stroke and is found relatively more often among younger AIS patients and in the aftermaths of minor trauma, such as sport [7-10]. Presence of CAD may impede access to the occlusion site and thereby negatively affect procedural performance metrics although patients with $C A D$ are on average younger and have lower rates of vascular risk factors. Due to these procedural challenges, CAD may be associated with worse outcome. Similar to the current situation with intravenous thrombolysis [11], data on the frequency, safety and efficacy of MT in CAD is relatively scarce. Previous reports have focused on comparisons between use of intravenous thrombolysis in comparison to MT [12]. There are no explicit recommendations on whether or not and how to treat patients with CAD with MT [13].

\section{Aim}

In the current study, we aimed to analyze procedural characteristics and radiological and functional outcome in patients with $\mathrm{LVO}$ and CAD who were treated with MT as part of routine care in Germany. Separate subgroup analyses were carried out for patients with anterior and posterior circulation stroke.

\section{Patients and methods}

This is a preplanned analysis insofar as a proposal with a detailed analysis plan had been handed in to the steering committee in July 2017. The proposal was approved by the steering committee of the German Stroke Registry Endovascular Treatment (GSR-ET) in 2017.

\section{Data availability}

The data that support the findings of this study are available from the corresponding author on reasonable request.

\section{Study population}

For the current study, we analyzed data from patients enrolled in the GSR-ET between April 2016 and April 2018. Details of the registry have been published previously $[3,14]$. Briefly, the GSR-ET is an ongoing industry-independent, open-label, academic, prospective, multicenter registry of adult patients (age $\geq 18$ years) with a diagnosis of AIS due to LVO treated by MT. Patients were recruited from 12 university hospitals and 13 municipal hospitals from all regions of Germany. All data entered into the registry were assessed locally by trained neuroradiologists and neurologists and underwent centrally coordinated quality checks for consistency, plausibility, and completeness [3, 15].

\section{Data extraction}

The following parameters were extracted from the database: baseline demographic, clinical, and radiological characteristics; procedural characteristics (e.g., number of passages, use of intra-arterial medication, treatment with an extracranial stent); technical outcome (reperfusion success measured by the modified Treatment in Cerebral Infarction [mTICI] scale); functional outcome (National Institutes of Health Stroke Scale [NIHSS] score on day 1 after thrombectomy and on hospital discharge; modified Rankin scale [mRS] score on day 1, at discharge, and on day 90); occurrence of complications; and the presumed etiology of the stroke according to the Trial of Org 10,172 in Acute Stroke Treatment (TOAST) classification [16]. A diagnosis of CAD was made by local investigators based on imaging results and findings from diagnostic tests according to current guidelines. Reperfusion success was defined as mTICI score $2 \mathrm{~b}-3$, favorable outcome as $\mathrm{mRS}$ score $\leq 2$. Intracranial hemorrhage was defined according to the ECASS II (European Cooperative Acute Stroke Study [part II]) definition and refers to symptomatic intracranial hemorrhage [17].

\section{Statistical analysis}

Continuous data are presented as medians and interquartile ranges (IQR), categorical data as percentages. For all parameters of interest, we primarily compared patients with CAD to those without CAD with additional stratification by location of the vessel occlusion (anterior vs. posterior circulation). In addition, we compared patients with CAD and occlusion of a vessel of the anterior circulation to patients with CAD and occlusion of a vessel of the posterior circulation. Baseline parameters, procedural characteristics, and reperfusion rates were compared between groups using Mann-WhitneyU-tests, Pearson chi-squared tests, and univariable ordinal and binary logistic regression analysis. For comparisons of functional outcome parameters (NIHSS score and $\mathrm{mRS}$ score), Mann-Whitney tests and univariable regression analyses were used for unadjusted analyses, and analysis of covariance and multivariable regression analyses for adjusted analyses (adjusted for possible confounders that were associated with the grouping variable in univariable analyses and that are known to affect outcome [age, gender, pre-stroke mRS score, medical history, prior antiplatelet and anticoagulation therapy, Alberta Stroke Program Early CT Score (ASPECTS), and 
location of occluded vessel]). For sensitivity analyses, procedural characteristics and reperfusion rates were also compared with adjustment for potential confounders. A two-sided $P$-value of.05 was considered statistically significant. Due to the exploratory nature of the analysis, no correction for multiple testing was applied.

\section{Informed consent and ethics approval}

Data collection for the GSR was centrally approved by the Ethics Committee of the Ludwig-Maximilians University, Munich (689-15) as the leading ethics committee. Further approval was obtained from local ethics committees or institutional review boards according to local regulations. In accordance with the institutional review board approval, no informed consent was required because no study-specific procedures were performed and data sampling from patients undergoing MT was already mandated by national law for quality control reasons.

\section{Results}

A total of 2589 patients with AIS and LVO treated with MT were included in the current analysis, of whom 62 (2.4\%) had a diagnosis of CAD.

\section{Baseline characteristics}

Baseline demographic, clinical, and radiological characteristics of patients with CAD and patients with other stroke etiologies are shown in Table 1. Patients with CAD were significantly younger (median [IQR]; 51 [4357] years vs. 76 [65-82] years, $P<.001$ ), less often female ( $25.8 \%$ vs. $50.9 \%, P<.001)$, and had less pre-stroke disability $(P<.001)$. In addition, patients with CAD had lower rates of vascular risk factors and were less often on antiplatelet $(17.7 \%$ vs. $33.5 \%, P=.009)$ or anticoagulation medication $(1.6 \%$ vs. $21.0 \%, P<.001)$ before stroke. Among patients without CAD, the most common etiology was cardioembolism (51.7\%), followed by largeartery atherosclerosis (25.9\%) and stroke of undetermined origin (17.5\%). Patients with CAD had lower median ASPECTS scores than patients with non-CAD etiologies (8 [6-10] vs. $9[7-10], P=.025)$, corresponding to larger baseline stroke volumes as detected by CT. Extracranial internal carotid artery stenosis was more common in patients with CAD than in those with other etiologies $(46.7 \%$ vs. $14.1 \%, P<.001)$.

Initial stroke symptom severity (NIHSS score), smoking status, treatment paradigm (direct-to-center vs. dripand-ship), delay between time of symptom onset/time last seen well and admission, and mTICI score before thrombectomy did not differ significantly between patients with and without CAD.

Of 28 patients with CAD and ICA occlusion, 5 patients had a concomitant M1 occlusion, 4 patients a concomitant M2 occlusion, and 1 patient a combined M1/M2 occlusion. Of 11 patients with CAD and VA occlusion, 5 patients had a concomitant BA occlusion, and 1 patient had a combined BA/PCA occlusion.

When comparisons were made within the subgroups of patients with stroke either in the anterior or posterior circulation, very similar between-group differences were present in both subgroups. The observed disparities in gender and in the proportion of internal carotid artery stenosis were only present within the anterior circulation subgroup and differences in pre-stroke medication did not reach statistical significance due to smaller sample sizes.

Limiting the comparisons to patients with either anterior or posterior circulation CAD, patients with anterior circulation CAD had significantly shorter median onsetto-admission times than patients with posterior circulation CAD (87 [54-171] min 262 [63-356] min, $P=.032$ ). The remaining baseline characteristics did not differ significantly between patients with anterior and posterior circulation CAD (Supplemental Table I).

\section{Procedural characteristics}

Intervention data and treatment time metrics are summarized in Table 2. Median time from admission to groin puncture and time from groin puncture to flow restoration was significantly longer in patients with $C A D$ than in patients without CAD (90 [56-126] min vs. 71 [46-104] $\mathrm{min}, P=.019$; and 98 [67-136] min vs. 70 [45$100] \mathrm{min}, P<.001$, respectively). Accordingly, total time from symptom onset (if known) to flow restoration was also significantly longer in the group of patients with CAD (322 [233-435] $\mathrm{min}$ vs. 245 [190-322] $\mathrm{min}$, $P<.001)$. Use of intra-arterial medication was approximately twice as frequent in the CAD group as in the non-CAD group $(34.4 \%$ vs. $15.6 \%, P<.001)$.

Within the subgroups of anterior or posterior circulation stroke only, differences in procedural characteristics according to presence or absence of CAD (additional intra-arterial medication, time intervals) were similar to those in the whole population. The only exception was the rate of intravenous thrombolysis, which was significantly higher in patients with CAD in anterior circulation occlusion $(80.0 \%$ vs. $55.8 \%, P<.001)$ but not in posterior circulation occlusion $(35.3 \%$ vs. $49.3 \%, P=$ .332). Focusing the analysis on patients with CAD only, we found that patients with anterior circulation occlusion were treated with intravenous thrombolysis significantly more often than patients with posterior circulation occlusion $(80.0 \%$ vs. $35.3 \%, P<.001$; Supplemental Table II).

All between-group differences pertaining to procedural characteristics were statistically non-significant in 
Table 1 Baseline characteristics

\begin{tabular}{|c|c|c|c|}
\hline & No $C A D, n=2527$ & CAD, $n=62$ & $P$ \\
\hline \multicolumn{4}{|l|}{ Demographic } \\
\hline Age - years, median (IQR), $\mathrm{n}$ & $76(65-82), n=2527$ & $51(43-57), n=62$ & $<.001$ \\
\hline Female, \% (n) & $50.9 \%(1286)$ & $25.8 \%(16)$ & $<.001$ \\
\hline \multicolumn{4}{|l|}{ Pre-stroke mRS, \% (n) } \\
\hline 0 & $68.0 \%(1652)$ & $95.2 \%(59)$ & $<.001$ \\
\hline 1 & $12.8 \%(310)$ & $0 \%(0)$ & \\
\hline 2 & $8.3 \%(201)$ & $3.2 \%(2)$ & \\
\hline 3 & $6.0 \%(145)$ & $1.6 \%(1)$ & \\
\hline 4 & $3.5 \%(86)$ & $0 \%(0)$ & \\
\hline 5 & $1.4 \%(35)$ & $0 \%(0)$ & \\
\hline Current smoking, \% (n) & $15.0 \%(332)$ & $24.1 \%(13)$ & .160 \\
\hline \multicolumn{4}{|l|}{ Clinical } \\
\hline $\mathrm{NIHSS}$, median (IQR), $\mathrm{n}$ & $15(10-19), n=2527$ & $14(7-17), n=62$ & .107 \\
\hline \multicolumn{4}{|l|}{ Medical history, \% (n) } \\
\hline Hypertension & $76.8 \%(1925)$ & $25.8 \%(16)$ & $<.001$ \\
\hline Diabetes mellitus & $21.3 \%(535)$ & $4.8 \%(3)$ & $<.001$ \\
\hline Dyslipidemia & $34.1 \%(854)$ & $17.7 \%(11)$ & .006 \\
\hline Atrial fibrillation & $41.9 \%(1049)$ & $1.6 \%(1)$ & $<.001$ \\
\hline \multicolumn{4}{|l|}{ Medication, \% (n) } \\
\hline Antiplatelet & $33.5 \%(822)$ & $17.7 \%(11)$ & .009 \\
\hline Anticoagulation & $21.0 \%(515)$ & $1.6 \%(1)$ & $<.001$ \\
\hline \multicolumn{4}{|l|}{ Etiology, \% (n) } \\
\hline Cardioembolism & $51.7 \%(1307)$ & - & - \\
\hline Dissection & - & $100.0 \%(62)$ & \\
\hline Large-artery atherosclerosis & $25.9 \%(654)$ & - & \\
\hline Small-vessel occlusion & $0.0 \%(1)$ & - & \\
\hline Stroke of other determined etiology & $4.8 \%(122)$ & - & \\
\hline Stroke of undetermined etiology & $17.5 \%(443)$ & - & \\
\hline Direct-to-center, \% (n) & $52.0 \%(1313)$ & $54.8 \%(34)$ & .701 \\
\hline Symptom onset known, \% (n) & $61.5 \%(1553)$ & $74.2 \%(46)$ & .047 \\
\hline Time from symptom onset to admission - min, median (IQR), n & 127 (59-206), $n=1553$ & $119(54-185), n=46$ & .728 \\
\hline Time from last-seen-well to admission - min, median (IQR), $\mathrm{n}$ & $357(202-685), n=974$ & $283(200-339), n=16$ & .163 \\
\hline \multicolumn{4}{|l|}{ Radiological } \\
\hline ASPECTS, median (IQR), $n$ & $9(7-10), n=1904$ & $8(6-10), n=37$ & .025 \\
\hline \multicolumn{4}{|l|}{ Occluded vessel, \% (n) } \\
\hline ICA & $24.5 \%(614)$ & $45.2 \%(28)$ & $<.001$ \\
\hline MCA, M1 & $56.7 \%(1423)$ & $29.0 \%(18)$ & $<.001$ \\
\hline MCA, M2 & $17.7 \%(444)$ & $19.4 \%(12)$ & .420 \\
\hline ACA & $2.5 \%(62)$ & $0.0 \%(0)$ & .403 \\
\hline BA & $10.2 \% 255)$ & $19.4 \%(12)$ & .032 \\
\hline VA & $1.7 \%(42)$ & $17.7 \%(11)$ & $<.001$ \\
\hline PCA & $2.2 \%(55)$ & $1.6 \%(1)$ & 1.000 \\
\hline \multicolumn{4}{|l|}{ mTICI before thrombectomy, \% (n) } \\
\hline 0 & $85.9 \%(2088)$ & $86.7 \%(52)$ & .801 \\
\hline
\end{tabular}


Table 1 Baseline characteristics (Continued)

\begin{tabular}{lll}
\hline & No CAD, $\mathbf{n}=\mathbf{2 5 2 7}$ & CAD, $\mathbf{n = 6 2}$ \\
\hline 1 & $4.8 \%(117)$ & $5.0 \%(3)$ \\
$2 \mathrm{a}$ & $2.1 \%(50)$ & $1.7 \%(1)$ \\
$2 \mathrm{~b}$ & $3.1 \%(75)$ & $5.0 \%(3)$ \\
3 & $4.2 \%(102)$ & $1.7 \%(1)$ \\
Extracranial ICA stenosis (> 70\%) & $14.1 \%(334)$ & $46.7 \%(28)$ \\
ipsilateral to intracranial vessel & & \\
occlusion, \% (n/n) & & $<.001$ \\
\hline
\end{tabular}

CAD stands for cervical artery dissection, IQR Interquartile range, mRS Modified Rankin scale, NIHSS National Institutes of Health Stroke Scale, ASPECTS Alberta Stroke Program Early CT Score, ICA Internal carotid artery, ACA Anterior cerebral artery, BA Basilar artery, VA Vertebral artery, PCA Posterior cerebral artery, $m$ TICI Modified Treatment in Cerebral Infarction

sensitivity analyses considering the differences in baseline characteristics as potential confounders.

\section{Reperfusion rates}

Reperfusion after MT quantified on the mTICI score did not differ significantly between patients with and without CAD (Table 3). No differences were observed within or between the subgroups of patients with anterior or posterior circulation stroke (Supplemental Table III).

\section{Adverse events}

Frequency of adverse events did not differ between patients with or without CAD, neither in the whole population nor in the subgroups confined to anterior or posterior circulation stroke. Rates of intracranial hemorrhage, vasospasm and recurrent stroke were below 5\% each (Table 4, Supplemental Table IV).

\section{Functional outcome}

In unadjusted analyses, follow-up NIHSS scores and $\mathrm{mRS}$ scores were nominally lower in patients with CAD as compared to patients without CAD indicating better recovery and less severe functional disability. After adjustment for differences in baseline characteristics (younger age and better pre-stroke functional status in CAD patients), these differences were no longer statistically significant. (Table 5 , Supplemental Table V). Patients with CAD had similarly high probabilities of a favorable outcome at 90 days irrespective of whether the

Table 2 Procedural characteristics

\begin{tabular}{|c|c|c|c|}
\hline & No $C A D, n=2527$ & CAD, $n=62$ & $P$ \\
\hline \multicolumn{4}{|l|}{ Intervention } \\
\hline Treatment with intravenous thrombolysis, \% (n) & $55.6 \%(1393)$ & $67.7 \%(42)$ & .069 \\
\hline \multicolumn{4}{|l|}{ Anesthesia, \% (n) } \\
\hline Conscious sedation & $30.2 \%(733)$ & $21.0 \%(13)$ & .281 \\
\hline Beginning with conscious sedation, switch to general anesthesia & $3.7 \%(90)$ & $4.8 \%(3)$ & \\
\hline Primary general anesthesia & $66.1 \%(1602)$ & $74.2 \%(46)$ & \\
\hline Number of passages, median (IQR), $\mathrm{n}$ & $2(1-3), n=2527$ & $2(1-3), n=62$ & .377 \\
\hline Intra-arterial medication, \% (n) & $15.6 \%(387)$ & $34.4 \%(21)$ & $<.001$ \\
\hline Additional heparin bolus given, \% ( $\mathrm{n}$ ) & $13.3 \%(303)$ & $27.1 \%(16)$ & .006 \\
\hline Concomitant stenting of > 70\% ipsilateral extracranial ICA stenosis (if present), \% (n) & $70.0 \%(231)$ & $64.3 \%(18)$ & .527 \\
\hline \multicolumn{4}{|l|}{ Time intervals } \\
\hline \multicolumn{4}{|l|}{ Known symptom onset } \\
\hline Time from symptom onset to flow restoration - min, median (IQR), n & $245(190-322), n=1553$ & $322(233-435), n=46$ & $<.001$ \\
\hline \multicolumn{4}{|l|}{ Unknown symptom onset } \\
\hline Time from last-seen-well to flow restoration - min, median (IQR), n & $520(352-833), n=974$ & $466(385-524), n=16$ & .657 \\
\hline \multicolumn{4}{|l|}{ Known and unknown symptom onset } \\
\hline Time from admission to i.v. thrombolysis - min, median (IQR), n & $21(-76-35), n=2527$ & $17(-71-30), n=62$ & .518 \\
\hline Time from admission to groin puncture - min, median (IQR), $\mathrm{n}$ & $71(46-104), n=2527$ & $90(56-126), n=62$ & .019 \\
\hline Time from groin puncture to flow restoration - min, median (IQR), n & $70(45-100), n=2527$ & $98(67-136), n=62$ & $<.001$ \\
\hline
\end{tabular}

$C A D$ stands for Cervical artery dissection, IQR Interquartile range, ICA Internal carotid artery 
Table 3 Reperfusion outcome

\begin{tabular}{ccll}
\hline & No CAD, $\boldsymbol{n}=\mathbf{2 5 2 7}$ & CAD, $\boldsymbol{n}=\mathbf{6 2}$ & $\boldsymbol{P}$ \\
\hline mTICI after thrombectomy, \% $(\mathbf{n})$ & & \\
0 & $9.0 \%(224)$ & $4.9 \%(3)$ & .116 \\
1 & $1.9 \%(48)$ & $0.0 \%(0)$ & \\
$2 \mathrm{a}$ & $5.7 \%(141)$ & $9.8 \%(6)$ & \\
2b & $35.6 \%(881)$ & $54.1 \%(33)$ & \\
3 & $47.7 \%(1182)$ & $31.1 \%(19)$ & \\
2b-3 & $83.3 \%(2063)$ & $85.2 \%(52)$ & .690
\end{tabular}

CAD stands for cervical artery dissection, $m$ TICI Modified Treatment in Cerebral Infarction

occlusion was in the anterior or posterior circulation (72.5\% vs. $66.7 \%, P=.856)$.

We performed an additional post-hoc exploratory analysis of the association between treatment with intravenous thrombolysis and outcome in patients with CAD. Adjusting for age, sex, and baseline NIHSS score, we found no significant association for the endpoints NIHSS score at $24 \mathrm{~h}$ and at discharge, and $\mathrm{mRS} \leq 2$ score at $24 \mathrm{~h}$ and at discharge ( $P$ values .707, .530, .252, and .760, respectively). Intravenous thrombolysis was associated with a higher probability of favorable functional outcome at 90 days (31 of 38 [82\%] versus 8 of 17 [47\%], odds ratio 20.8, 95\% confidence interval 2.6 to $168 ; P=.004$ ).

\section{Discussion}

CAD is a rare challenge in MT for AIS with LVO found in 62 of $2589(2.4 \%)$ patients in our large, multicenter registry of academic and non-academic centers mirroring real-world practice in Germany.

With respect to demographics and in line with previous studies, CAD patients in our registry were younger and had lower rates of cardiovascular comorbidities [10]. These characteristics should entail a good prognosis. However, imaging revealed more severe early ischemic changes (as assessed by ASPECTS score) in CAD patients. Lower ASPECTS score may mirror the lack of time to develop cerebral circulation collaterals in time, as arterial dissection is an acute process. Worse collaterals status is independently associated with larger infarct size [18]. Lower ASPECTS may also mirror the higher frequency of ipsilateral carotid stenosis in anterior circulation CAD patients possibly impairing perfusion pressure. Moreover, the median time delay between symptom onset and flow restoration was longer in CAD patients, which is also associated with worse outcome [19]. This longer time delay was at least in part attributable to a longer time period between groin puncture and flow restoration. The latter may reflect that intervention in CAD patients is more demanding, particularly since patients with CAD were younger than patients without CAD and were therefore less likely to have tortuous vessels or high atherosclerotic burden meaning that the CAD itself presumably challenged the interventionalist. The finding that CAD patients received intra-arterial medication more frequently, too, supports the notion of a more demanding intervention in CAD patients.

Nevertheless, despite more severe early ischemic changes on imaging and longer procedural time periods, both radiological (reperfusion) and adjusted functional outcome did not differ significantly between patients with and without CAD. Adverse events did not occur more frequently in CAD patients. The disadvantageous imaging and time parameters may have been balanced by the younger age of CAD patients which is a major indicator for better outcome [20, 21]. Previous data did not appear to be as positive for AIS patients with CAD as found by our real-world data. A post-hoc analysis of the MR CLEAN trial intervention arm and MR CLEAN Registry revealed reperfusion rates that appeared to be lower than those found in our registry $(47-58 \%$ in MR CLEAN versus $79-84 \%$ in our registry) [22]. Other studies examined smaller study populations or did not compare anterior and posterior circulation stroke.

Besides a longer time delay between groin puncture and flow restoration, we also observed a significantly longer delay between admission and groin puncture in patients with CAD. Possible explanations for this

Table 4 Adverse events

\begin{tabular}{|c|c|c|c|}
\hline & No $C A D, n=2527$ & CAD, $n=62$ & $P$ \\
\hline Any adverse events, \% (n) & $13.4 \%(334)$ & $12.9 \%(8)$ & 1.000 \\
\hline Device malfunction & $0.3 \%(8)$ & $0.0 \%(0)$ & $\stackrel{a}{a}$ \\
\hline Dissection/perforation & $2.8 \%(70)$ & $4.8 \%(3)$ & a \\
\hline Clot migration/embolism & $3.1 \%(78)$ & $4.8 \%(3)$ & a \\
\hline Intracerebral hemorrhage & $2.2 \%(55)$ & $0.0 \%(0)$ & a \\
\hline Vasospasm & $2.6 \%(65)$ & $4.8 \%(3)$ & $\stackrel{a}{a}$ \\
\hline New persistent neurological deficit & $0.0 \%(2)$ & $0.0 \%(0)$ & a \\
\hline New transient neurological deficit & $0.0 \%(1)$ & $0.0 \%(0)$ & a \\
\hline
\end{tabular}

${ }^{a}$ No $P$-value estimated due to low numbers. CAD stands for cervical artery dissection 
Table 5 Functional outcome

\begin{tabular}{|c|c|c|c|c|}
\hline & No $C A D, n=2527$ & $C A D, n=62$ & $\begin{array}{l}\text { Parameter estimate } \\
(95 \% \mathrm{Cl})^{\mathrm{a}}\end{array}$ & $\begin{array}{l}P_{\text {crude }} \\
P_{\text {adj }} \\
\end{array}$ \\
\hline \multicolumn{5}{|l|}{ After $24 \mathrm{~h}$} \\
\hline NIHSS after $24 \mathrm{~h}$, median (IQR), n & $10(4-20), n=2527$ & $6.5(2-15), n=62$ & $\begin{array}{l}b, c \\
-1.39(-4.85-2.06)^{d}\end{array}$ & $\begin{array}{l}.010^{\mathrm{b}} \\
.428^{\mathrm{d}}\end{array}$ \\
\hline \multicolumn{5}{|l|}{ mRS score after 24 h, \% (n) } \\
\hline 0 & $2.8 \%(60)$ & $10.5 \%(6)$ & \multirow{7}{*}{$\begin{array}{l}0.60(0.13-1.07)^{b} \\
-0.04(-0.71-0.63)^{d}\end{array}$} & \multirow{7}{*}{$\begin{array}{l}.013^{b} \\
.901^{d}\end{array}$} \\
\hline 1 & $6.1 \%(133)$ & $12.3 \%(7)$ & & \\
\hline 2 & $8.7 \%(190)$ & $7.0 \%(4)$ & & \\
\hline 3 & $13.1 \%(286)$ & $14.0 \%(8)$ & & \\
\hline 4 & $18.0 \%(391)$ & $14.0 \%(8)$ & & \\
\hline 5 & $50.5 \%(1100)$ & $42.1 \%(24)$ & & \\
\hline 6 & $0.8 \%(17)$ & $0.0 \%(0)$ & & \\
\hline$\leq 2$ & $17.6 \%(383)$ & $29.8 \%(17)$ & $\begin{array}{l}-0.69(-1.27--0.11)^{b} \\
0.35(-0.60-1.30)^{d}\end{array}$ & $\begin{array}{l}.020^{\mathrm{b}} \\
.472^{\mathrm{d}}\end{array}$ \\
\hline \multicolumn{5}{|l|}{ At discharge } \\
\hline NIHSS at discharge, median (IQR), $n$ & $6(2-13), n=2527$ & $3(0-10), n=62$ & $\begin{array}{l}b, c \\
0.23(-3.05-3.52)^{d}\end{array}$ & $\begin{array}{l}.003^{b} \\
.889^{d}\end{array}$ \\
\hline \multicolumn{5}{|l|}{ mRS score at discharge, \% (n) } \\
\hline 0 & $8.2 \%(202)$ & $20.0 \%(12)$ & \multirow{7}{*}{$\begin{array}{l}0.95(0.50-1.40)^{b} \\
0.26(-0.34-0.86)^{d}\end{array}$} & \multirow{7}{*}{$\begin{array}{l}<.001^{b} \\
.403^{d}\end{array}$} \\
\hline 1 & $12.0 \%(296)$ & $21.7 \%(13)$ & & \\
\hline 2 & $11.6 \%(286)$ & $10.0 \%(6)$ & & \\
\hline 3 & $13.6 \%(336)$ & $16.7 \%(10)$ & & \\
\hline 4 & $17.0 \%(420)$ & $10.0 \%(6)$ & & \\
\hline 5 & $21.7 \%(534)$ & $16.7 \%(10)$ & & \\
\hline 6 & $15.8 \%(390)$ & $5.0 \%(3)$ & & \\
\hline$\leq 2$ & $31.8 \%(784)$ & $51.7 \%(31)$ & $\begin{array}{l}-0.83(-1.34--0.32)^{b} \\
0.20(-0.55-0.96)^{d}\end{array}$ & $\begin{array}{l}.002^{b} \\
.599^{d}\end{array}$ \\
\hline \multicolumn{5}{|l|}{ After 90 days } \\
\hline \multicolumn{5}{|l|}{ mRS score after 90 days, \% (n) } \\
\hline 0 & $11.7 \%(260)$ & $18.2 \%(10)$ & \multirow{7}{*}{$\begin{array}{l}1.06(0.59-1.53)^{b} \\
0.29(-3.5-0.94)^{d}\end{array}$} & \multirow{7}{*}{$\begin{array}{l}<.001^{b} \\
.372^{d}\end{array}$} \\
\hline 1 & $14.3 \%$ (318) & $32.7 \%(18)$ & & \\
\hline 2 & $10.4 \%(232)$ & $20.0 \%(11)$ & & \\
\hline 3 & $12.4 \%(275)$ & $5.5 \%(3)$ & & \\
\hline 4 & $12.7 \%(283)$ & $9.1 \%(5)$ & & \\
\hline 5 & $10.2 \%(226)$ & $9.1 \%(5)$ & & \\
\hline 6 & $28.4 \%(632)$ & $5.5 \%(3)$ & & \\
\hline$\leq 2$ & $36.4 \%(810)$ & $70.9 \%(39)$ & $\begin{array}{l}-1.45(-2.04--0.86)^{b} \\
-0.74(-1.59-0.10)^{d}\end{array}$ & $\begin{array}{l}<.001^{\mathrm{b}} \\
.086^{\mathrm{d}}\end{array}$ \\
\hline
\end{tabular}

Parameter estimates and 95\% confidence intervals derived from univariate general linear (NIHSS score), ordinal (mRS distributions), or binary (dichotomized mRS score) regression models; the reference category is formed by patients with CAD. ${ }^{b}$ Unadjusted models ${ }^{c}$ No parameter estimate derived from from Mann-Whitney test. ${ }^{d}$ Analysis adjusted for age, gender, pre-stroke modified Rankin scale score, medical history, prior medication, Alberta Stroke Program Early CT Score, and location of occluded vessel. CAD stands for cervical artery dissection; IQR, interquartile range; mRS, modified Rankin scale; NIHSS, National Institutes of Health Stroke Scale

observation may include a) less streamlined diagnostic and decision making processes in patients with younger age, i.e. a demographic characteristic not usually associated with a diagnosis of stroke, or b) therapeutic uncertainty once a decision of ischemic stroke due to $\mathrm{LVO}$ in the presence of CAD is made.

Additional post-hoc analyses indicated that bridging intravenous thrombolysis may be associated with better 
functional outcome at 90 days in patients with CAD undergoing MT. While the non-randomized design, the lack of effect in respect to the other functional outcome endpoints, and modest sample size limit the external validity of this observation, it provides reassurance for thrombolytic treatment in this clinical context; further studies are, however, warranted.

To our knowledge, our study is the first to analyze MT in stroke patients with CAD in the posterior circulation separately. Although limited by a relatively small number of patients $(n=17)$, our data provide evidence that support the use of MT for eligible AIS patients with CAD and occlusion within the posterior circulation. In our sample, successful reperfusion (mTICI grade $2 \mathrm{~b}-3$ ) could be achieved in $17 / 17$ patients, adverse events were rare, and most patients (66.6\%) were functionally independent after 3 months.

All this should encourage the treating physicians to perform MT in patients with AIS due to LVO in both the anterior as well as the posterior circulation.

We are aware of the following limitations. First, although our data were derived from a large prospective registry, the number of patients with CAD included in the analysis $(N=62)$ was only moderate which could have limited the study's ability to detect existing between-group differences (type 2 error). Given the low prevalence of CAD among stroke patients in general, collaborative efforts combining data from multiple registries might be helpful in the future. Second, although data checks were conducted at each hospital to ensure complete documentation, we cannot rule out selection bias entirely. Third, management of CAD patients occurred according to current guidelines; however, there was no common protocol for the management of CAD patients executed identically by all hospitals. We therefore do not have detailed data on the order of different maneuvers performed (for example stenting first versus embolectomy first) or the precise peri-interventional antiplatelet regimen used. Registries collecting these information in greater detail will be of benefit for further analyses in the future. Fourth, information about the location of CAD was limited to anterior versus posterior circulation; no information was available regarding the affected vessel or the extent of the dissection. Fifth, we did not assess pc-ASPECTS in posterior circulation occlusion, although pc-ASPECTS would have given a more precise marker of early ischemic changes in these patients. Finally, only presence (or absence) of carotid stenosis was known but not its exact severity in terms of degree, its pathophysiologic origin, and whether or not it was causal to the stroke. However, we assume that the majority of relatively young patients with CAD would not have severe pre-existing atherosclerotic ICA stenosis.

\section{Conclusion}

In conclusion, our analysis demonstrates that AIS patients with $\mathrm{LVO}$ due to CAD represent a distinct patient population that poses specific therapeutic challenges for the clinician and the interventionalist. In spite of more severe early ischemic changes on imaging, longer procedure times and higher requirement for concomitant intra-arterial medical treatment, functional outcome does not appear worse than in patients without CAD in both the anterior and posterior circulation. Future studies should aim to identify modifiable contributors to treatment delay in patients with CAD.

\section{Supplementary Information}

The online version contains supplementary material available at https://doi. org/10.1186/s42466-021-00119-y.

Additional file 1 Supplemental Table I Baseline characteristics in subgroups of patients with anterior or posterior circulation occlusion. Supplemental Table II Procedural characteristics in subgroups of patients with anterior or posterior circulation occlusion. Supplemental Table III Reperfusion outcomes in subgroups of patients with anterior or posterior circulation occlusion. Supplemental Table IV Adverse events in subgroups of patients with anterior or posterior circulation occlusion. Supplemental Table V Functional outcome in subgroups of patients with anterior or posterior circulation occlusion.

\section{Acknowledgments}

LS is participant in the $\mathrm{BIH}$-Charite Clinical Scientist Program funded by the Charité - Universitätsmedizin Berlin and the Berlin Institute of Health.

\section{Authors' contributions}

CHN designed and conceived the study. LS performed the statistical analyses and wrote the first draft of the manuscript. All authors contributed to patient recruitment and data collection, were involved in interpretation of the data, critically reviewed and edited the manuscript for intellectual content and approved the final version of the manuscript.

\section{Funding}

None

\section{Availability of data and materials}

The data that support the findings of this study are available from the corresponding author on reasonable request.

\section{Declarations}

Ethics approval and consent to participate

Data collection for the GSR was centrally approved by the Ethics Committee of the Ludwig-Maximilians University, Munich (689-15) as the leading ethics committee. Further approval was obtained from local ethics committees or institutional review boards according to local regulations.

\section{Consent for publication}

In accordance with the institutional review board approval, no informed consent was required because no study-specific procedures were performed and data sampling from patients undergoing MT was already mandated by national law for quality control reasons.

\section{Competing interests}

LS reports lecture fees from Daiichi Sankyo, outside of the submitted work FF reports consulting fees from Eppdata, outside of the submitted work. GCP reports consulting fees or fees as lecturer from Bayer, Boehringer Ingelheim, Bristol-Myers Squibb and Pfizer, all outside of the submitted work. 
GT reports consulting fees or fees as lecturer from Acandis, Bayer, Boehringer Ingelheim, Bristol-Myers Squibb, Pfizer, Portola, Stryker, all outside of the submitted work

ME reports grants from Bayer and fees paid to the Charité from Bayer, Boehringer Ingelheim, BMS/Pfizer, Daiichi Sankyo, Amgen, GSK, Sanofi, Covidien, and Novartis, all outside of the submitted work $\mathrm{CHN}$ reports consulting and lecture fess from Boehringer Ingelheim, W.L. Gore and Associates, Bristol-Myers Squibb, Pfizer, and Abbott, all outside of the submitted work.

The others have no disclosures.

\section{Author details}

${ }^{1}$ Klinik und Hochschulambulanz für Neurologie, Charité - Universitätsmedizin Berlin, corporate member of Freie Universität Berlin, Humboldt-Universität zu Berlin, Berlin, Germany. ${ }^{2}$ Berlin Institute of Health (BIH), Berlin, Germany. ${ }^{3}$ Center for Stroke Research Berlin (CSB), Charité - Universitätsmedizin, Berlin, Germany. ${ }^{4}$ DZNE (German Center for Neurodegenerative Diseases), Partner Site Berlin, Berlin, Germany. ${ }^{5}$ Department of Neuroradiology, Charité Universitätsmedizin Berlin, corporate member of Freie Universität Berlin, Humboldt-Universität zu, Berlin, Germany. ${ }^{6}$ Universitätsklinikum Hamburg-Eppendorf, Klinik und Poliklinik für Neuroradiologische Diagnostik und Intervention, Martinistr 52, 20246 Hamburg, Germany. ${ }^{7}$ German Center for Neurodegenerative Diseases (DZNE), Bonn, Bonn, Germany. ${ }^{8}$ Department of Neurology, Division of Vascular Neurology, University Hospital Bonn, Bonn, Germany. ${ }^{9}$ Universitätsklinikum Hamburg-Eppendorf, Klinik und Poliklinik für Neurologie, Martinistr. 52, 20246 Hamburg, Germany. ${ }^{10}$ DZHK (German Center for Cardiovascular Research), Partner Site Berlin, Berlin, Germany. ${ }^{11}$ Department of Neurology, Charite - Campus Benjamin Franklin, Hindenburgdamm 30, 12200 Berlin, Germany.

\section{Received: 4 March 2021 Accepted: 16 March 2021}

Published online: 03 May 2021

\section{References}

1. Powers, W. J., Rabinstein, A. A., Ackerson, T., Adeoye, O. M., Bambakidis, N. C., Becker, K., et al. (2019). Guidelines for the early Management of Patients with Acute Ischemic Stroke: 2019 update to the 2018 guidelines for the early Management of Acute Ischemic Stroke: A guideline for healthcare professionals from the American Heart Association/American Stroke Association. Stroke, 50(12), e344-e418. https://doi.org/10.1161/STR. 0000000000000211.

2. Turc, G., Bhogal, P., Fischer, U., Khatri, P., Lobotesis, K., Mazighi, M., ... Fiehler, J. (2019). European stroke organisation (ESO) - European Society for Minimally Invasive Neurological Therapy (ESMINT) guidelines on mechanical Thrombectomy in acute Ischaemic StrokeEndorsed by stroke Alliance for Europe (SAFE). European Stroke Journal, 4(1), 6-12. https://doi.org/10.1177/23 96987319832140.

3. Wollenweber, F. A., Tiedt, S., Alegiani, A., Alber, B., Bangard, C., Berrouschot, J., ... Fiehler, J. (2019). Functional outcome following stroke Thrombectomy in clinical practice. Stroke, 50(9), 2500-2506. https://doi.org/10.1161/STROKEA HA.119.026005.

4. Sporns, P. B., Hanning, U., Schwindt, W., Velasco, A., Minnerup, J., Zoubi, T., ... Niederstadt, T. U. (2017). Ischemic stroke: What does the histological composition tell us about the origin of the thrombus? Stroke, 48(8), 22062210. https://doi.org/10.1161/STROKEAHA.117.016590.

5. Tiedt, S., Herzberg, M., Kupper, C., Feil, K., Kellert, L., Dorn, F., et al. (2020). Stroke etiology modifies the effect of endovascular treatment in acute stroke. Stroke, 51(3), 1014-1016.

6. Berkhemer, O. A., Fransen, P. S., Beumer, D., van den Berg, L. A., Lingsma, H. F., Yoo, A. J., et al. (2015). A randomized trial of intraarterial treatment for acute ischemic stroke. The New England Journal of Medicine, 372(1), 11-20. https://doi.org/10.1056/NEJMoa1411587.

7. Schlemm, L., Nolte, C. H., Engelter, S. T., Endres, M., \& Ebinger, M. (2017). Cervical artery dissection after sports - an analytical evaluation of 190 published cases. European Stroke Journal, 2(4), 335-345. https://doi.org/10.11 77/2396987317720544

8. Bejot, Y., Daubail, B., Debette, S., Durier, J., \& Giroud, M. (2014). Incidence and outcome of cerebrovascular events related to cervical artery dissection: The Dijon stroke registry. International Journal of Stroke, 9(7), 879-882. https://doi.org/10.1111/ijs.12154.
9. Lee, V. H., Brown Jr., R. D., Mandrekar, J. N., \& Mokri, B. (2006). Incidence and outcome of cervical artery dissection: A population-based study. Neurology, 67(10), 1809-1812. https://doi.org/10.1212/01.wnl.0000244486.30455.71.

10. Debette, S., Compter, A., Labeyrie, M. A., Uyttenboogaart, M., Metso, T. M., Majersik, J. J., ... Bousser, M. G. (2015). Epidemiology, pathophysiology, diagnosis, and management of intracranial artery dissection. Lancet Neurology, 14(6), 640-654. https://doi.org/10.1016/\$1474-4422(15)00009-5.

11. Berge, E., Whiteley, W., Audebert, H., GMD, M., Fonseca, A. C., Padiglioni, C., ... Turc, G. (2021). European stroke organisation (ESO) guidelines on intravenous thrombolysis for acute ischaemic stroke. European Stroke Journal, O(0), 2396987321989865.

12. Lin, J., Liang, Y., \& Lin, J. (2019). Endovascular therapy versus intravenous thrombolysis in cervical artery dissection-related ischemic stroke: A metaanalysis. Journal of Neurology, 267(6), 1585-1593. https://doi.org/10.1007/ s00415-019-09474-y.

13. Powers, W. J., Rabinstein, A. A., Ackerson, T., Adeoye, O. M., Bambakidis, N. C., Becker, K., ... Tirschwell, D. L. (2019). Guidelines for the early Management of Patients with acute ischemic stroke: 2019 update to the 2018 guidelines for the early Management of Acute Ischemic Stroke: A guideline for healthcare professionals from the American Heart Association/American Stroke Association. Stroke, 50(12), e344-e418. https://doi.org/10.1161/STR. 0000000000000211.

14. Alegiani, A. C., Dorn, F., Herzberg, M., Wollenweber, F. A., Kellert, L., Siebert, E., ... Thomalla, G. (2019). Systematic evaluation of stroke thrombectomy in clinical practice: The German stroke registry endovascular treatment. International Journal of Stroke, 14(4), 372-380. https://doi.org/10.1177/17474 93018806199.

15. Flottmann, F., Broocks, G., Faizy, T. D., McDonough, R., Watermann, L., DebChatterji, M., et al. (2020). Factors associated with failure of reperfusion in endovascular therapy for acute ischemic stroke: A multicenter analysis. Clinical Neuroradiology.

16. Adams Jr., H. P., Bendixen, B. H., Kappelle, L. J., Biller, J., Love, B. B., Gordon, D. L., \& Marsh 3rd, E. E. (1993). Classification of subtype of acute ischemic stroke. Definitions for use in a multicenter clinical trial. TOAST. Trial of org 10172 in acute stroke treatment. Stroke, 24(1), 35-41. https://doi.org/10.11 61/01.STR.24.1.35.

17. Hacke, W., Kaste, M., Fieschi, C., von Kummer, R., Davalos, A., Meier, D., ... Trouillas, P. (1998). Randomised double-blind placebo-controlled trial of thrombolytic therapy with intravenous alteplase in acute ischaemic stroke (ECASS II). Second European-Australasian acute stroke study investigators. Lancet, 352(9136), 1245-1251. https://doi.org/10.1016/S0140-6736(98)08020-9.

18. Singer, O. C., Berkefeld, J., Nolte, C. H., Bohner, G., Reich, A., Wiesmann, M., ... Liebeskind, D. S. (2015). Collateral vessels in proximal middle cerebral artery occlusion: The ENDOSTROKE study. Radiology, 274(3), 851-858. https://doi.org/10.1148/radiol.14140951.

19. Saver, J. L., Goyal, M., van der Lugt, A., Menon, B. K., Majoie, C. B., Dippel, D. W., et al. (2016). Time to treatment with endovascular Thrombectomy and outcomes from ischemic stroke: A meta-analysis. JAMA, 316(12), 1279-1288. https://doi.org/10.1001/jama.2016.13647.

20. Singer, O. C., Haring, H. P., Trenkler, J., Nolte, C. H., Bohner, G., Reich, A., ... for the ENDOSTROKE Study Group (2013). Age dependency of successful recanalization in anterior circulation stroke: The ENDOSTROKE study. Cerebrovascular Diseases, 36(5-6), 437-445. https://doi.org/10.1159/0003 56213.

21. Goyal, M., Menon, B. K., van Zwam, W. H., Dippel, D. W., Mitchell, P. J., Demchuk, A. M., et al. (2016). Endovascular thrombectomy after large-vessel ischaemic stroke: A meta-analysis of individual patient data from five randomised trials. Lancet, 387(10029), 1723-1731. https://doi.org/10.1016/ S0140-6736(16)00163-X.

22. Compagne, K. C. J., Goldhoorn, R. B., Uyttenboogaart, M., van Oostenbrugge, R. J., van Zwam, W. H., van Doormaal, P. J., et al. (2019). Acute endovascular treatment of patients with ischemic stroke from intracranial large vessel occlusion and Extracranial carotid dissection. Frontiers in Neurology, 10, 102. https://doi.org/10.3389/fneur.2019.00102.

\section{Publisher's Note}

Springer Nature remains neutral with regard to jurisdictional claims in published maps and institutional affiliations. 